\title{
Superficial scrapings from breast tumors is a source for biobanking and research purposes
}

\author{
Ran $\mathrm{Ma}^{1}$, Irma Fredriksson ${ }^{2,3}$, Govindasamy-Muralidharan Karthik', Gregory Winn ${ }^{5}$, Eva Darai-Ramqvist ${ }^{5}$, \\ Jonas Bergh ${ }^{1,4}$ and Johan Hartman ${ }^{1,5}$
}

Breast cancer is a unique tumor disease in terms of the stringent requirement of predictive biomarker assessments. As recommended by current international guidelines, the established markers consist of estrogen receptor (ER), progesterone receptor, human epidermal growth factor and Ki67, and are primarily analyzed by immunohistochemistry. However, new diagnostic methods based on microarray or next-generation sequencing on DNA and mRNA level are gaining ground. These analyses require fresh-frozen tumor tissue that is generally not available from tumors $<10 \mathrm{~mm}$ in diameter, comprising almost $25 \%$ of all resected breast cancer at our department. We here present a simple and standardized method to generate material from small tumors without risking the histopathological examination.

Furthermore, we show that the quality of this material is sufficient for subsequent analysis on mRNA, DNA, and epigenetic level. We were also able to use this method for isolation and expansion of cancer stem cells from the majority of tumors. Consequently, researches can be provided with clinically relevant material for translational studies. In conclusion, this method opens up a new possibility for usage of valuable fresh tumor material for research purposes, biobanking, and next-generation sequencing.

Laboratory Investigation (2014) 94, 796-805; doi:10.1038/labinvest.2014.65; published online 28 April 2014

KEYWORDS: mammary neoplasia; pathology; superficial scrapings; tissue biobanking; tumor-initiating cells

Breast cancer is a complex disease comprising different subtypes, each with its own biological characteristics and prognosis. ${ }^{1}$ During the past decade, scientific breakthroughs have offered new methods to characterize breast cancer according to molecular subtypes and to predict therapeutic response with an accuracy not seen before. ${ }^{2}$ However, these evolving diagnostic methods will expect clinical pathology laboratories to update tissue handling and analytical pipelines to adjust to new demands from both patients and clinicians. Consequently, fresh tissue biobanking from resected breast cancers specimens is a central and given method at most University Hospitals. ${ }^{3,4}$

Preserved tissue material may be used for additional clinical investigations on DNA, mRNA, and biomarker protein levels after routine histopathological assessments, ${ }^{5-7}$ and is a unique source for future research projects. Two different biobanking methods are used for this purpose, although the later is by many institutions considered to be too costly and complicated:

(1) Preservation of tissue through standard paraffin embedding of formalin-fixated material (FFPE). As a consequence of recent technological advancements, FFPE blocks can now be used as a source for whole-genome DNA sequencing, at least in the experimental setting. ${ }^{8}$

(2) Rapid freezing of tissue pieces and storage in a lowtemperature freezer. This is the preferred method for long-term storage and the only method to preserve mRNA efficiently, protein and DNA for subsequent analysis. ${ }^{9,10}$ Consequently, FFPE-based analytical methods should only be considered as an alternative when frozen samples are not available.

With most research biobanks being based on snap-frozen tumor pieces, there are two evident sampling problems: first,

\footnotetext{
${ }^{1}$ Departments of Oncology and Pathology, Cancer Center Karolinska, Karolinska Institutet, Stockholm, Sweden; ${ }^{2}$ Departments of Molecular Medicine and Surgery, Karolinska Institutet, Stockholm, Sweden; ${ }^{3}$ Departments of Breast and Endocrine Surgery, Karolinska Institutet, Stockholm, Sweden; ${ }^{4}$ Radiumhemmet - Karolinska Oncology, Karolinska University Hospital, Stockholm, Sweden and ${ }^{5}$ Departments of Clinical Pathology and Cytology, Karolinska University Hospital, Stockholm, Sweden Correspondence: Dr J Hartman, MD, PhD, Departments of Clinical Pathology and Cytology, Karolinska University Hospital, Karolinska Vägen, 17176 Stockholm, Sweden. E-mail: johan.hartman@ki.se
} 
tumor pieces contain varying ratios of cancer cells to stromal cells. Although experimental evidence points at important roles for the tumor stroma in cancer progression, there is a substantial risk of stromal non-malignant contamination in analytical assessments of breast cancer cells. ${ }^{11,12}$ For instance, certain morphological subtypes show considerable infiltration of lymphocytes or normal mammary glands, whereas other breast cancers show a solid growth pattern. This dilution effect may affect the global gene expression patterns and mutation-allele frequencies of tumors. It has been speculated that analysis of purified cancer cells would predict therapy response and prognosis more accurately than whole tumor tissue. ${ }^{13}$ Second, small tumors $(<10 \mathrm{~mm}$ in diameter) comprise a major obstacle as all available tumor tissue is typically required for routine diagnostics. The advent of mammographic screening programs and an increasing number of patients treated with neoadjuvant chemotherapy also leads to decreasing diameters of the resected tumors. Hence, it is a general concern that biobanks will soon only consist of samples from larger tumors and thereby have an inherited bias. New alternative methods to perform biobanking from small tumor lesions are highly desirable.

Here, we show that superficial scrapings from breast tumors are sufficient as biobanking resource for investigations on DNA and RNA levels. Moreover, we show that scraping material can be enriched for cancer cells with stem cell capacity. This opens up a new possibility for translational research from clinical material. Consequently, small tumors could be used in biobank and research procedures without jeopardizing the routine histopathological assessment.

\section{MATERIALS AND METHODS Clinical Material}

All tissues and scrapings were obtained at Karolinska University Hospital after approved and signed informed consents and in compliance with standardized surgical procedures. Fresh biopsies and scrapings from primary breast cancer were immediately processed for biobanking and cell purification. Permits were obtained from the regional ethics board at Karolinska Institutet in Stockholm and from the biobank at Karolinska University Hospital.

\section{Cytological Assessments of Smears From Superficial Scrapings}

For cytological assessments, smears from superficial scrapings were air-dried on the glasses and fixed for $15 \mathrm{~min}$ in methanol. The fixed slides were then stained for $5 \mathrm{~min}$ in May-Grünwald solution diluted with an equal volume of distilled water. Finally, the smears were transferred into diluted Giemsa solution with 9 volume of distilled water without washing for another $30 \mathrm{~min}$. The slides were washed in the water and dried. The stained smears were observed under the light microscope.

\section{Cryopreservation and Generation of Mammospheres From Scrapings}

Superficial scrapings were performed on freshly opened breast tumors by scraping 3-10 times around the edge of the margin of the tumor. The scrapings were immediately processed either for biobanking or for the initiation of mammospheres in selective medium as described elsewhere. ${ }^{14}$ Cells were quickly transferred into either an empty cryotube directly by wiping the blade on the tube mouth for the low-temperature biobanking or transferred into a prepared cryotube with freezing media (10\% DMSO + 90\% serum) for the step-freezing cryopreservation of viable cells. For mammosphere isolation, scrapings were washed two times in PBS. After centrifugation, the final cell pellets were re-suspended in the selective medium. For the first 3 days of culturing, clumps were seen to form. To generate proper mammospheres, clumps were collected by gentle centrifugation at $100 \mathrm{~g}$ for $5 \mathrm{~min}$, followed by trypsinization and dissociation, single cells were seeded in 6-well plates at the density of 5000-20000 cells per ml. Mammospheres could be observed after 5 days in culture. Importantly, only cultures with proliferating/growing mammospheres were further studied.

\section{Proliferation and Viability Assays}

For proliferation assays, single cells were obtained and then plated at clonogenic density 1000-3000 viable cells per well in 48-well plates (Corning Costar, Cambridge, MA, USA) as triplicates to form mammospheres. Plates were incubated for 10 days; newly formed mammospheres were optically counted and documented. The mammosphere-forming efficiency was calculated as percentages (number of mammospheres per well per number of cells seeded per well) $\times 100$.

For viability assays, cryopreserved vials of scrapings were taken from liquid nitrogen and quickly thawed in water bath at $54^{\circ} \mathrm{C}$. Cells were washed in PBS two times and transferred into the selective medium to recover. After culturing for 3 days, debris was removed by step centrifugation, and the clumps were enzymatically dissociated into single cells to form mammospheres.

\section{Immunofluorescence Staining and Image Analysis}

Around 40-50 mammospheres were spun onto glass slides, fixed in 4\% paraformaldehyde for $20 \mathrm{~min}$ and blocked in PBS containing $0.05 \%$ Tween and $2.5 \%$ bovine serum albumin (all from Sigma-Aldrich, St Louis, MO, USA) for $45 \mathrm{~min}$. Slides were rinsed in PBS for three times in between antibody incubations. 4',6-Diamidino-2-phenylindole (DAPI) (Life Technologies, Carlsbad, CA, USA) 1:1000 was used to visualize and distinguish nuclei of the cells. Slides were mounted with VECTASHIELD ${ }^{\circledR}$ mounting medium (Vector Laboratories, Burlingame, CA, USA) and ready for analysis. We used the following primary antibodies to characterize the immunophenotype of the mammospheres: rabbit polyclonal anti-human CD44 1:100 (HPA005785; Sigma, St Louis, MO, 
USA), mouse monoclonal anti-human CD24-biotin (clone: 32D1) 1:50 (10231; StemCell Technologies, Vancouver, BC, Canada), rabbit polyclonal anti-human aldehyde dehydrogenase 1 (ALDH1) 1:100 (ab23375; Abcam, Cambridge, UK), mouse monoclonal anti-human cytokeratin (clone: AE1/AE3) 1:50 (M3515; Dako, Carpinteria, CA, USA), mouse monoclonal anti-human smooth muscle actin (SMA) (clone: 1A4) 1:50 (M0851; Dako), mouse monoclonal antihuman epithelial cell adhesion molecule (EpCAM) (clone: VU1D9) 1:50 (ab98003; Abcam). For PKH26 staining, cells were incubated with $10^{-7} \mathrm{M}$ PKH26 (Sigma-Aldrich) for $5 \mathrm{~min}$ and then grown in suspension for 7 consecutive days to enable the formation of new spheres. Stained samples were analyzed and digital images were taken under a computerized fluorescence microscope equipped with CCD camera. Pictures were processed with Photoshop CS5.

\section{DNA, RNA Extraction, and Quality Control}

Snap frozen scrapings and paired bulk tumors were gently thawed on ice after collection from $-80^{\circ} \mathrm{C}$ and directly processed for nucleic acid isolation. RNA extractions were performed on both samples according to the manufacturer's instructions (RNAeasy Mini Kit; Qiagen, Valencia, CA, USA). DNA was isolated from thawed scrapings by using the QIAamp DNA Mini Kit (Qiagen). RNA concentration and size distribution were assessed by Nanodrop 1000 measurement and determining UV 260/280 absorbance ratios on RNA Nano LabChips (Agilent Technologies, Santa Clara, CA, USA) processed on the Agilent 2100. RNA integrity number (RIN) score was generated for each trace by using 2100 Expert Software (Agilent Technologies). The maximum RIN score is 10. There is no numerical scoring algorithm for this program. DNA quantification and integrity were examined by Nanodrop 1000 (Nanodrop Technologies, Wilmington, DE, USA) measurement and running gel electrophoresis.

\section{CDNA Synthesis and Real-Time PCR}

Cell pellets from frozen scrapings and corresponding bulk tumors were collected. After RNA isolation as described above, cDNA synthesis was performed with the First Strand System (Nordic Bioservice AB, Stockholm, Sweden) according to the standard protocol. Quantitative real-time PCR (RT-PCR) was performed with Power SYBR green ${ }^{\mathbb{R}}$ detection system on the FAST 7500 Real-Time PCR System (Applied Biosystems, Foster City, CA, USA). The thermal cycler was programmed as follows: for holding stage $-95^{\circ} \mathrm{C}$ for $10 \mathrm{~min}$ and for cycling stage $-95^{\circ} \mathrm{C}$ for $15 \mathrm{~s}$, and $60^{\circ} \mathrm{C}$ for $50 \mathrm{~s}$ repeated for 40 cycles in total. The following primers were used: estrogen receptor $\alpha(E R \alpha)$ (F: 5'-GCTACGAAGTG GGAATGATGAAAG- $3^{\prime}$; R: $5^{\prime}$-TCTGGCGCTTGTGTTTCA AC-3'); PR (F: $5^{\prime}$-TGCCCAGCATGTCGCCTTAG-3'; R: $5^{\prime}$-A ACAGCATCCAGTGCTCTCAC-3'); $\alpha$-SMA (F: $5^{\prime}$-TCCCCG GGACTAAGACGGGAATC- $3^{\prime}$; R: $5^{\prime}$-TGGGAGCATCGTCCC CAGCA- $3^{\prime}$ ); PDGFR $\beta$ (F: $5^{\prime}$-GAGACTGTTGGGCGAAGG TTA-3'; R: 5'-GGATGGCTGGGTGGTCACT-3'); EрCAM
(F: 5'-GCGACTTTTGCCGCAGCTCA-3'; R: 5'-TTTGGCAG CCAGCTTTGAGCAA- $3^{\prime}$ ); and $18 \mathrm{~S}$ (F: $5^{\prime}$-GCTTAATTTGA CTCAACACGGGA-3'; R: $5^{\prime}$-AGCTATCAATCTGTCAATCCT GTC- $3^{\prime}$ ) as a reference gene. Fold changes of each gene at the mRNA level over control $18 \mathrm{~S}$ were calculated by $\Delta \mathrm{Ct}$ according to the standard curve method.

\section{FACS Analysis Procedures for the CD44 + /CD24 - Population}

Single cells trypsinzed from fresh breast cancer scrapings were blood lysed and washed with ice-cold PBS containing $1 \%$ FCS, and resuspended in the same washing buffer at the density of $10^{6}$ cells per $200 \mu \mathrm{l}$. PE-conjugated mouse anti-human CD24 (clone: SN3), FITC-conjugated mouse anti-human CD44 (clone: MEM-85 were added to the cell suspensions at the concentrations as recommended by the manufacturer's instructions. Tubes were well mixed and then incubated on ice in the dark for $30 \mathrm{~min}$. The labeled cells were washed three times to stop staining and resuspended in the 500-700 $\mu$ PBS containing 1\% FCS. A measure of $1 \mu \mathrm{l}$ propidium iodide was added in each tube for the viability. Cells were analyzed on a LSRII flow cytometer (BD Biosciences, San Jose, CA, USA).

Separation of the ALDH + Population by FACS Analysis Primary cells obtained from freshly dissociated breast cancer scrapings were isolated with their ALDH enzymatic activities by use the ALDEFLUOR Kit (StemCell Technologies). In general, around 50000 cells were suspended in $500 \mu \mathrm{l}$ ALDEFLUOR assay buffer with $5 \mu \mathrm{l}$ ALDH substrate (BAAA) and incubated critically at $37^{\circ} \mathrm{C}$ for $40 \mathrm{~min}$. As the negative background control, a spare of an aliquot cell from the same biopsy were co-treated with ALDH substrate and an equal volume of an ALDH inhibitor diethylaminobenzaldehyde (DEAB) during incubation. The positive sorting gate was identified using the negative control cells stained with propidium iodide.

\section{DNA Methylation and Pyrosequencing}

DNA from the scrapings of two patients with different ER $\alpha$ status was isolated using QIAamp DNA Mini Kit (Qiagen). DNA quality was verified and quantified using Nanodrop 1000. Bisulfite conversion was performed with $1 \mu \mathrm{g}$ of extracted DNA using EpiTect Bisulfite Kit (Qiagen). Two PyroMark CpG assays: (1) Hs_ESR1_02_PM (161 bp, with the following sequence to analyze 5'-CGTGTCCCC GCAGGGCAGAAGGCTCAGAAACCGGCGGGC- $3^{\prime}$ covering four CpGs); (2) Hs_ESR1_01_PM (114 bp, with the following sequence to analyze $5^{\prime}$-CGACAGCTGCGGCGGCGGG T- $3^{\prime}$ covering four CpGs) were ordered. PyroMark PCR Kit was used to amplify the specific sequence of DNA for pyrosequencing. PyroMark Q24 was used for pyrosequencing along with the PyroMark Gold Q24 reagents. All the steps were followed according to the standard protocol mentioned by the manufacturer. 


\section{Statistical Analyses}

Two-tailed $t$-test was performed to test statistical significance for the comparison of RT-PCR data between bulk tumor and scrapings. $P<0.05$ was considered significant and nonsignificant results were represented as NS.

\section{RESULTS}

\section{Enrichment of Breast Cancer Cells by Superficial Scrapings}

We obtained the tumor specimens directly from breast surgery ( $<3 \mathrm{~h}$ from surgery in room temperature) as a part of the routine gross-pathological procedure. Within the majority of cases, the localization of the tumors within the specimen was marked by a needle at specimen X-ray. By horizontal sectioning through the breast specimen, the tumor was identified and bisected. At the Department of Breast and Endocrine Surgery, Karolinska University Hospital, tumors of varying size were resected (median diameter $18 \mathrm{~mm}$ in year 2011). Patients with locally advanced disease or aggressive tumor characteristics generally obtain neoadjuvant chemotherapy that frequently causes reduction of tumor volume, and for some subgroups even pathological complete remission. Within this study, we focused on tumors $<10 \mathrm{~mm}$ in diameter. We performed superficial scrapings and collection of epithelial cells through a stringent protocol immediately after exposure of the tumor surface (Figure 1). In parallel, cytological smear material from the scrapings was examined by a cytologist (Figures $2 \mathrm{a}$ and $\mathrm{b}$ ). The microscopic

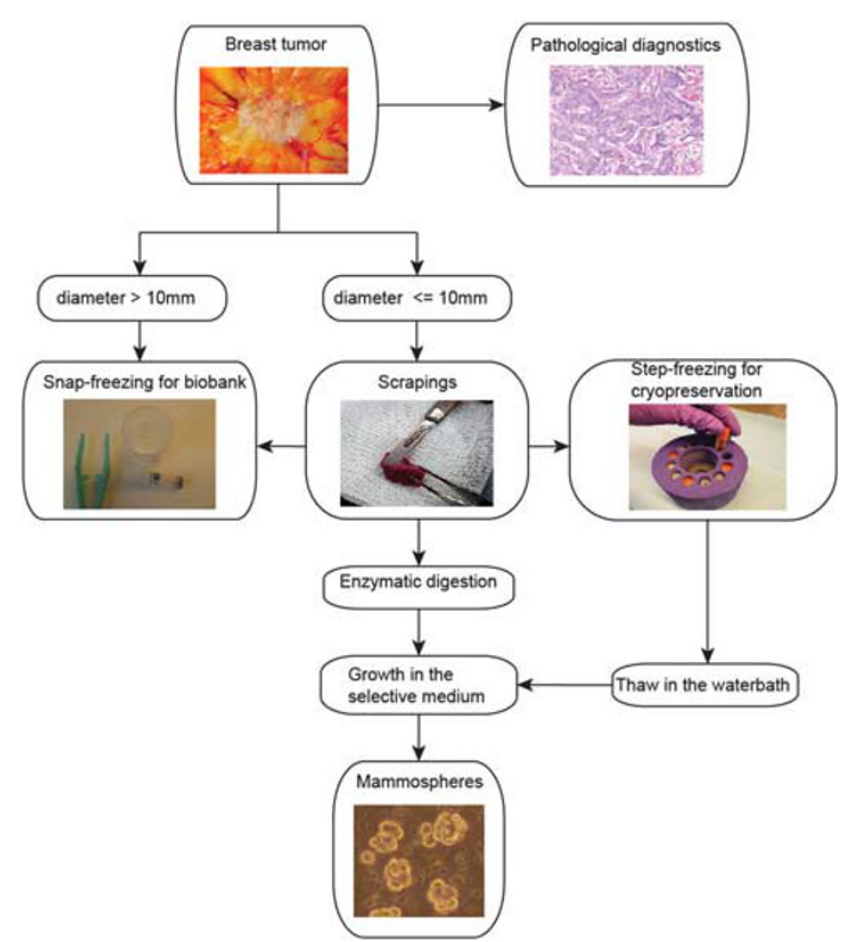

Figure 1 Project overview. The flow chart illustrates the process used to evaluate superficial scrapings for biobanking and research purposes. evaluation of smears from eight out of eight patients revealed a majority (>95\%) of the cells consisting of cancer cells. In many tumors, lymphocytes, dense stroma, or adipose tissue constitutes a large part of the tumor. Our finding indicates an enrichment of cancer cells in the scrapings as the corresponding primary tumor area consisted of $75-80 \%$ cancer cells as assessed by hematoxylin-eosin staining of tissue sections (Figures $2 \mathrm{c}$ and $\mathrm{d}$ ).

\section{Generation of Cancer Stem-Like Cells from Superficial Scrapings}

Cancer cells with stem cell characteristics (CSCs) are a minority within most tumors, but are responsible for maintaining tumor growth over a long time and in initiating metastasis. ${ }^{15}$ CSCs are phenotypically characterized by the cell-surface markers CD44 + CD24 - , the functional marker $\mathrm{ALDH} 1^{\text {High }}$ and the fluorescent marker $\mathrm{PKH} 26$, which labels quiescent cells. ${ }^{16-18}$ Hence, high expression of CSC-phenotypic markers in vivo correlates with poor prognosis. ${ }^{17,19}$ Another way to isolate CSC is based on the functionality to form multicellular spheres in low-attachment plates in vitro, normally inducing apoptosis of differentiated cancer cells. Consequently, there is a strong enrichment of cancer cells with CSC-phenotypic markers in mammospheres. ${ }^{20,21}$

By scraping 3-10 times with scalpels on the bisected tumor surface, we obtained a cell suspension that was directly transferred into conditional stem cell medium for 3 days in non-adherent serum-free conditions. This procedure stimulates the cancer cell population while removing contaminating fibroblasts and debris. ${ }^{22,23}$ During the process, some cells will form clumps and the cell cultures are then enzymatically dissociated to form single cells (Figure 1 and Materials and methods section). Within 5 days, mammospheres are obtained. By this process, we were able to isolate mammospheres from all four major molecular subtypes (Figure 3a). Further, all cultures could be propagated and expanded for at least three generations. In each generation, single cells from dissociated mammospheres were transferred into new culture wells and then left to proliferate into new multicellular spheres. We assessed the efficiency of mammosphere formation from superficial scrapings. From 13 of the 20 scrapings that generated cells, we successfully generated secondary mammosphere cultures (Table 1). As expected, mammospheres were identified as $\mathrm{CD} 44+/ \mathrm{CD} 24-$, $\mathrm{ALDH} 1^{\mathrm{High}}, \mathrm{EpCAM}+$, and PKH26 + (Figure 3b), indicating an enrichment of CSC within the mammospheres. The stem cell phenotype was further characterized by fluorescentactivated cell sorting (FACS). Around 30\% of cells were $\mathrm{ALDH}^{\text {High }}$ (Figure $3 \mathrm{c}$ ) and $40-50 \%$ of cells were CD44+ / CD24 - (Figure 3d). We also generated adherent cancer cells directly by plating scrapings and growing them in differentiating cell medium (Figure $3 \mathrm{e}$ ). These cells were also possible to expand, although with a certain risk of contamination from fibroblasts. 

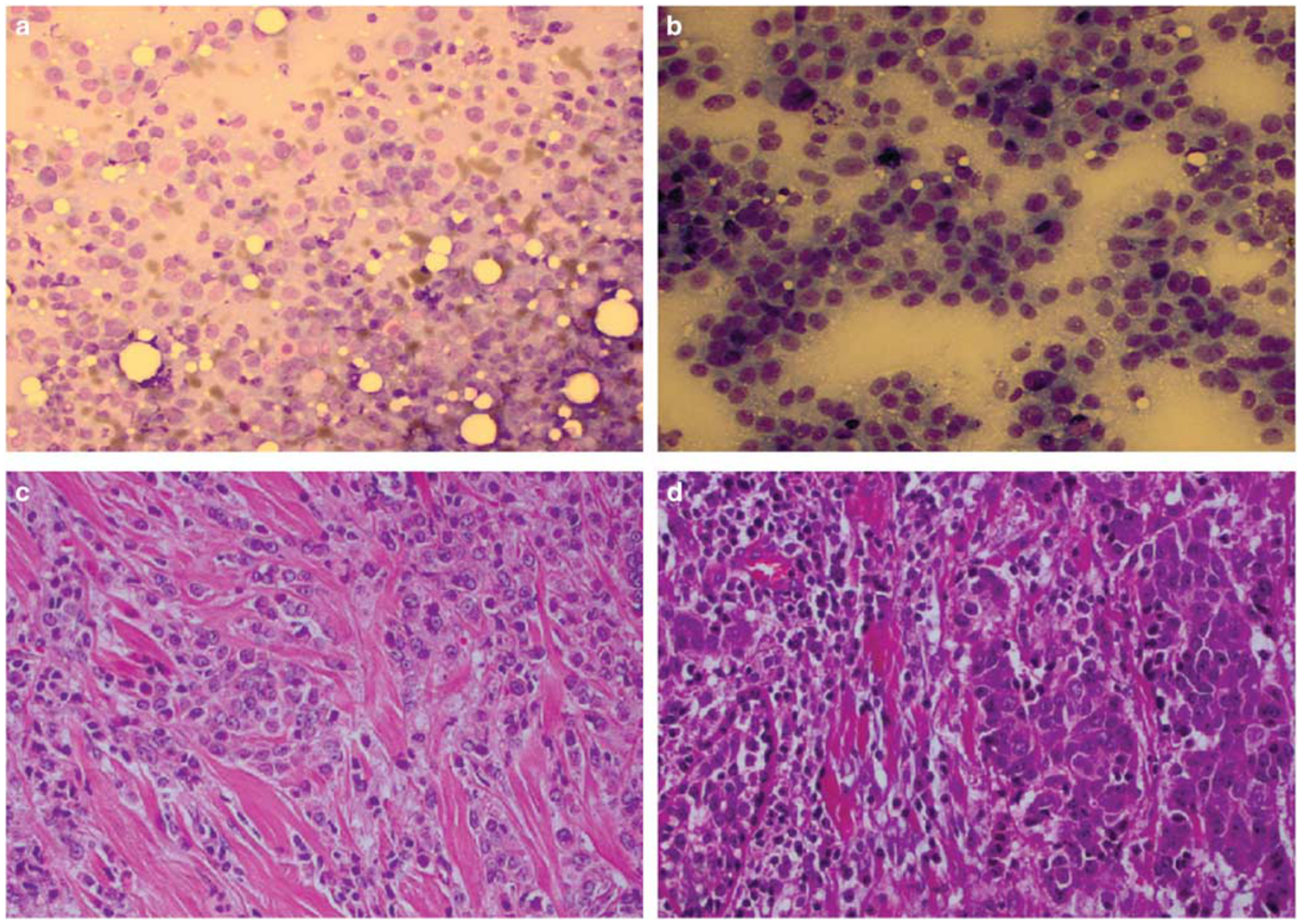

Figure 2 May-Grünwald (MGG)-stained smears of cells from superficial scrapings ( $\mathbf{a}$ and $\mathbf{b}$ ) and corresponding bright-field images of tissue sections from two patients stained by hematoxylin-eosin (c and $\mathbf{d})$, original magnification, $\times 200$.

We performed freezing of superficial scrape in cryopreservative cell medium by snap-freezing in liquid nitrogen. One week later, the cultures were thawed and the viability was assessed (Supplementary Figure S1). We found that the thawed material was a source for mammosphere initiation. However, not all frozen samples were able to form new mammospheres. Consequently, we think that the manual scraping procedure is critical for obtaining enough cells for live cell biobanking. Alternatively, only a subset of cancers is able to generate mammospheres that are able to survive cryopreservation.

\section{Low-Temperature Stored Scraping Material is Sufficient for DNA and RNA Analysis}

Scrapings from tumors were directly transferred to cryopreservative tubes. The tubes were put immediately into liquid nitrogen and stored in $-80{ }^{\circ} \mathrm{C}$. After 1 week, samples were thawed and DNA or mRNA was isolated. To assess the RNA efficiency in the scrapings versus paired tumors for future biobanking purpose, the RNA quality was analyzed on the basis of microchip gel electrophoresis and quantified by measuring the total absorbance. The partitioned regions of electropherograms from scrapings and frozen tumor pieces showed distinct ribosomal peaks and the RIN value ranged between 5.8 and 7.8 (Figure 4a and Supplementary Figures S2a and b), indicating no severe degradation during the biobanking procedure. Furthermore, the concentration spectral data showed good RNA yields from all the scrapings and of acceptable purity (Table 2), thereby enabling usage of scraping material for gene expression assays.

To investigate whether the superficial scraping can truly represent the gene expression pattern of the original breast cancers, we also prepared mRNA and reverse-transcribed cDNA from both scrapings and corresponding breast cancer biopsies. Based on the results from RT-PCR (Figure 4b), scrapings shared similar expression patterns of $E R \alpha$ and progesterone receptor $(P R)$ mRNA compared with their parental tumors; however the expression level varied among $2-5$-fold changes (one exception). To characterize the cellular content of superficial scrapings further, we examined $\alpha-S M A$, platelet-derived growth factor receptor- $\beta$ (PDGFR $\beta$ ) (genes overexpressed in fibroblasts), EPCAM (epithelial-specific gene) expressions using RT-PCR. Interestingly, the expression level of $\alpha-S M A$ and PDGFR $\beta$ mRNA was 6-10 times lower in 

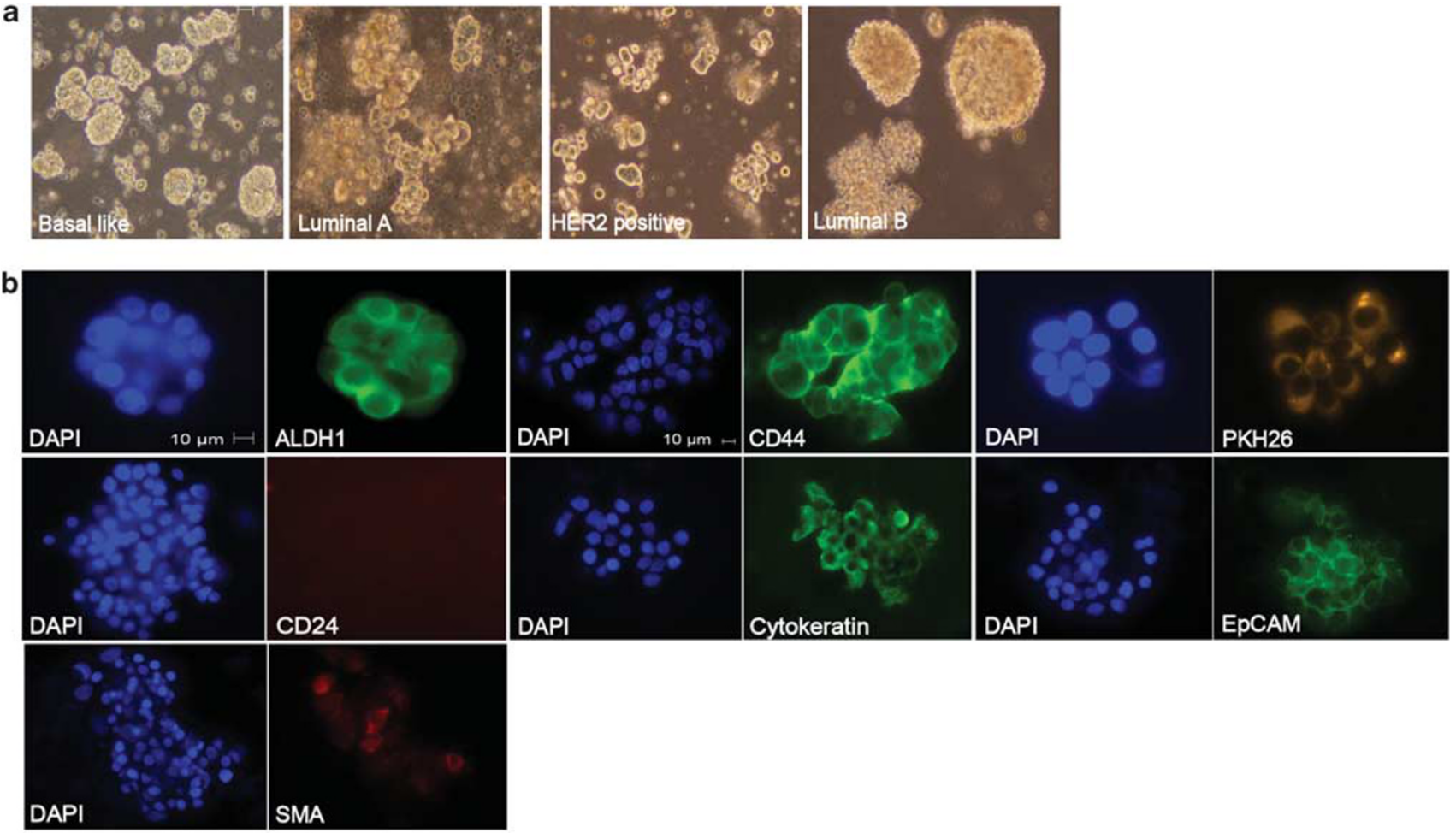

C

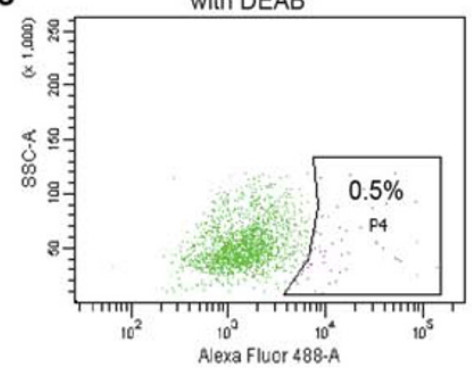

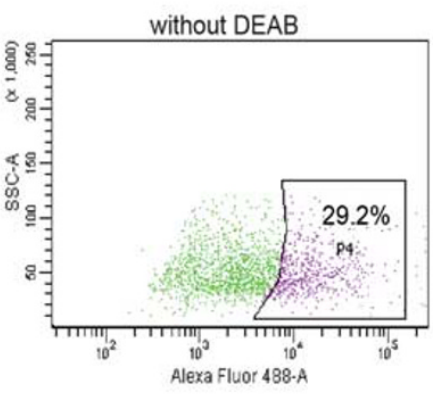

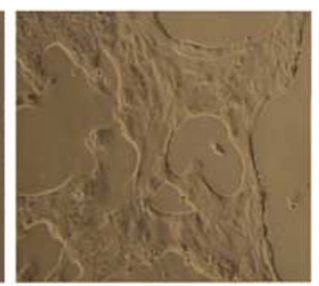

d

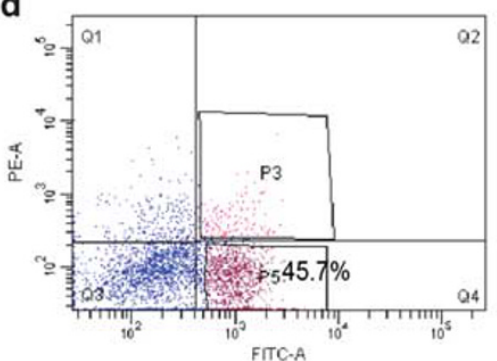

e

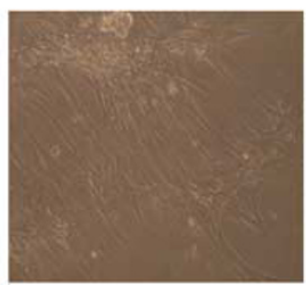

Figure 3 Breast cancer scrapings were cultured in a selective medium for 10 days and generated stable mammospheres. (a) Representative bright-field images of morphology from different breast cancer subtypes. Original magnification, $\times 400$. (b) Immunofluorescence imaging of mammospheres stained with different cell markers: Cytoplasmic ALDH1 ${ }^{\text {High }}$ (green) counterstained with 4',6-diamidino-2-phenylindole (DAPI) (blue); cell surface CD44 + (green) counterstained with DAPI; PKH26-staining + (yellow/brown) counterstained with DAPI; myofibroblast marker smooth muscle actin (SMA) (red) counterstained with DAPI; cell surface CD24 - (red) counterstained with DAPI; epithelial marker cytokeratin (green) counterstained with DAPI; cancer stem cell (CSC)/carcinoma marker epithelial cell adhesion molecule (EpCAM) (green) counterstained with DAPI. (c) Subpopulations of cells expressing prospective CSC markers in mammospheres. Fluorescent-activated cell sorting (FACS) profiles of diethylaminobenzaldehyde (DEAB) control (left) and aldehyde dehydrogenase (ALDH) staining (right) of single cells stained with ALDEFLUOR. (d) FACS analysis to characterize CD44 and CD24 expression level of single cells dissociated from scraping-generated mammospheres. (e) Representative bright-field images of differentiated cancer cells from mammospheres. Spheres from two patients (left and right) were incubated in the selective medium with $5 \%$ fetal bovine serum (FBS) and induced a switch from non-adherent to a spindle-like, adherent cell phenotype. The experiment was repeated two times with similar results (totally five patients). Original magnification, $\times 400$. 
Table 1 MFE from patient-derived cancer cells ${ }^{a}$

\begin{tabular}{|c|c|c|c|c|}
\hline Patient no. & Efficiency (\%) & Ki67 (\%) & $\begin{array}{l}\text { Tumor size } \\
\left(\mathrm{mm}^{2}\right)\end{array}$ & Subtypes \\
\hline 1 & 5.40 & 16 & $28 \times 22$ & Luminal A \\
\hline 2 & 2.00 & 30 & $24 \times 23$ & Luminal B (HER2 - ) \\
\hline 3 & 2.60 & 60 & $30 \times 22$ & HER2 \\
\hline 4 & 7.75 & 10 & $25 \times 16,11 \times 8$ & Luminal B $(\mathrm{HER} 2+)$ \\
\hline 5 & 3.50 & 12 & $19 \times 17$ & Luminal $\mathrm{A}$ \\
\hline 6 & 5.17 & 4 & $9 \times 8$ & Luminal A \\
\hline 7 & 5.14 & 42 & $50 \times 50$ & Luminal B (HER2 - ) \\
\hline 8 & 3.00 & 60 & $24 \times 20$ & Luminal B (HER2 - ) \\
\hline 9 & 4.42 & 5 & $22 \times 23$ & Luminal B $(\mathrm{HER} 2+)$ \\
\hline 10 & 4.38 & 25 & $20 \times 12$ & Luminal B (HER2 - ) \\
\hline 11 & 8.80 & 2 & $52 \times 42$ & Luminal B $(\mathrm{HER} 2+)$ \\
\hline 12 & 3.60 & 20 & $32 \times 24$ & Luminal B (HER2 - ) \\
\hline 13 & 3.65 & 15 & $46 \times 35$ & Luminal $\mathrm{A}$ \\
\hline
\end{tabular}

Abbreviation: MFE, mammosphere-forming efficiency.

In total, 1000-3000 cells from dissociated primary breast cancer scrapings were plated in triplicates onto 48-well for 7 days. MFE was calculated as dividing the number of mammospheres formed by the original number of single cells seeded as percentage.

${ }^{a}$ MFE from 13 patient-derived cancer cells associated with different subtypes and Ki67 scores.

scrapings versus bulk tumors. EpCAM expressed similar amounts, at the range of 2 fold-changes, in both groups. This gene expression pattern most probably reflects the enriched fraction of cancer cells in the superficial scrapings versus the bulk tumor.

DNA concentration and quality from scraping material were initially checked by Nanodrop 1000 after DNA extraction. Superficial scrapings provided decent amount of DNA yield (Table 3 ). Absorbance $A_{260 / 280}$ and $A_{260 / 230}$ ratio indicated proper and acceptable purity. Again, the purity and together with the integrity of DNA, were evaluated by running gel electrophoresis (Figure 5a). Intact genomic DNA appeared as compact, high-molecular-weight bands with slightly low-molecular-weight smears. Taken together, this analysis indicates that DNA isolated from lowtemperature stored scrapings is sufficient for next-generation sequencing (NGS).

In addition, we sought to investigate the usage of scraping material for epigenetic studies. DNA extracted from the scrapings of two patients, one being ER $\alpha+(90 \%)$ and the other ER $\alpha-(0 \%)$ were used to analyze the $E R \alpha$ methylation status using pyrosequencing technique. Two CpG assays were used to cover eight $\mathrm{CpGs}$ sites across the $E R \alpha$ promoter region (Supplementary Figures S3 and S4). We observed $E R \alpha$ promoter methylation in the $E R \alpha$ - patient using both CpG assays (Figure 5b). DNA from superficial scrapings of the bulk tumor retained the key methylation signatures, which correlates to the gene expression of the bulk tumor itself.

\section{DISCUSSION}

Tissue preservation by biobanking in low-temperature freezers is a valuable resource for additional diagnostic investigations on tumor material. This is especially evident in breast cancer diagnostics where microarray assessment of global gene expression is emerging as an additional diagnostic tool for therapy prediction. Of economical reasons, multigene expression assays are still provided only to a few patients, but the situation might soon change as the costs are decreasing. During the past years several large-scale whole genome and targeted sequencing procedures (NGS) have uncovered genetic rearrangements and actionable mutations in many patients. Most probably, NGS-based DNA profiling will soon be used as more or less routine methods in breast cancer diagnostics. As fresh-frozen tumor material is the golden standard for these methods, routine biobanking will be of utmost importance in the coming years. Fresh-frozen tumor samples also have the potential to play an essential part in the translational cancer research.

We here report a simple and robust technique for isolation of cancer cells from small breast tumors. At the Department of Pathology, Karolinska University Hospital, we currently should be able to include approximately $60 \%$ of all cancers in the biobank through standard biopsies taken from fresh tumor tissue. By including superficial scrapings from tumors considered too small for routine biobanking, we would then be able to increase the biobank inclusion to $85 \%$ of resected tumors. As we report here, this material can be successfully used for investigations on the DNA and RNA level. However, it cannot be used for current proteomics investigations that still require material in the $20-50 \mu \mathrm{g}$ range. As with freshfrozen biopsies that can be confirmed by frozen sectioning, similarly, cancer cell content of superficial scrapings can be confirmed with cytology before freezing. Most interestingly, it appears that superficial scraping results in an enrichment of cancer cells. The mechanism behind such an effect is unknown. We speculate that cancer cells are more easily detached from the tumor surface compared with stromal cells, thereby causing a higher cancer cell/stromal ratio in the scraping material. It should also be pointed out that the correlation of gene expression analysis based on frozen biopsies and scraping material is unconfirmed. As we show, superficial scrapings will be enriched in cancer cells and contain higher epithelial to stromal ratio compared with tumor biopsies. The predictive and prognostic role of enriched cancer cells for studies on mRNA level needs to be confirmed in prospective studies. If correctly performed, scraping by scalpel from the bisected tumor surface will generate cells from a large tumor area. However, as with ordinary biopsies, there will always be a risk of sampling bias as a consequence of the intratumoral heterogeneity. 

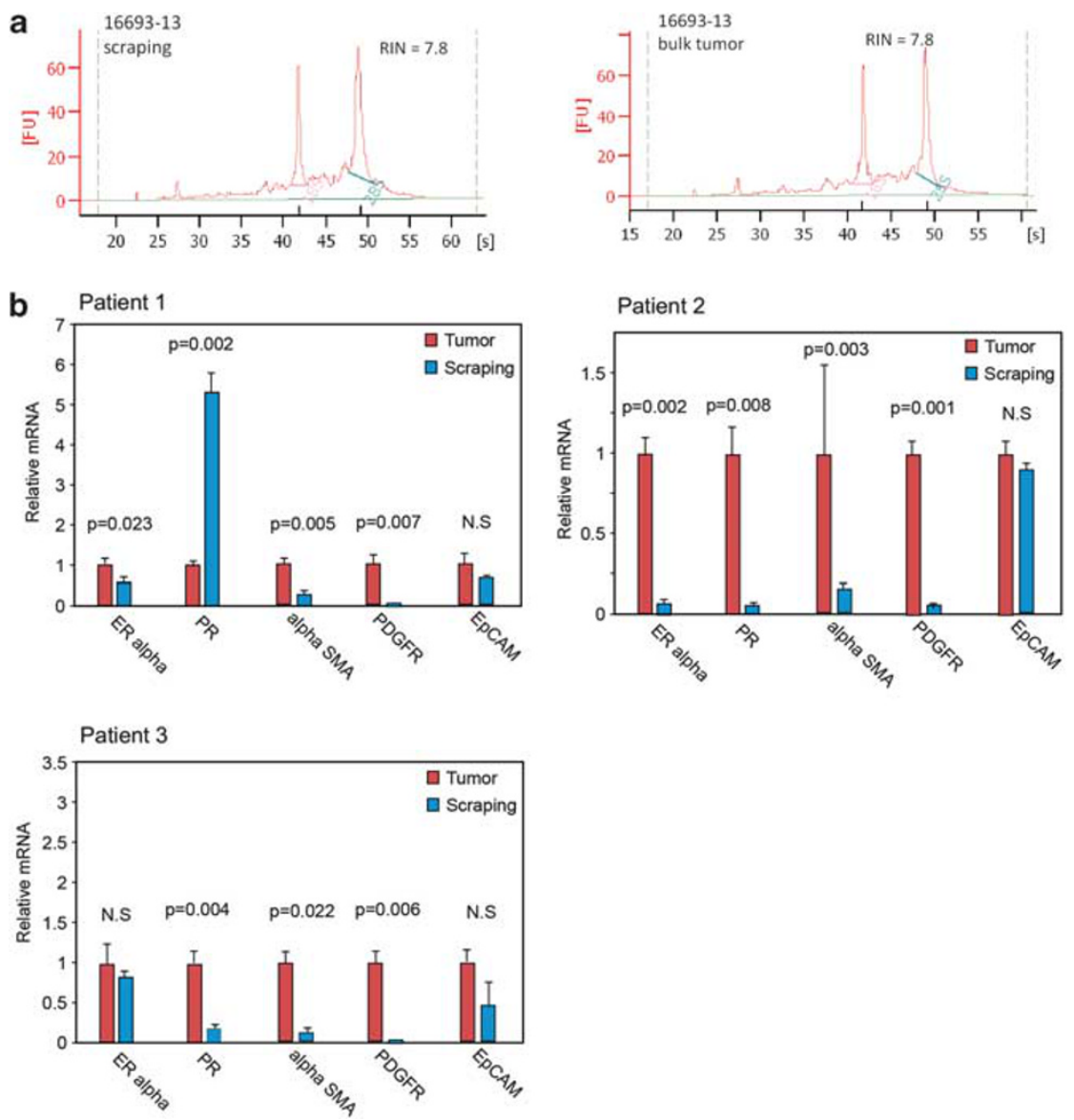

Figure 4 RNA quality control and validation. RNA was isolated from snap-frozen scrapings or biopsies from frozen tumor pieces. (a) RNA quality was assessed in the plot format by using the RNA 6000 Nano LabChip Kit and the 2100 Bioanalyzer, and the corresponding RNA integrity numbers (RINs) for each extraction is illustrated. (b) Real-time PCR (RT-PCR) was performed to compare the expression pattern of estrogen receptor- $\alpha$ (ER $\alpha$ ), progesterone receptor (PR), $\alpha$-smooth muscle actin $(\alpha-S M A)$, platelet-derived growth factor receptor $(P D G F R)$ and EpCAM between bulk tumor and scrapings from three breast cancer patients. Student's $t$-test was used to calculate the significance $(P<0.05)$ between the samples.

\section{Table 2 RNA quality control ${ }^{\mathrm{a}}$}

\begin{tabular}{llllcr}
\hline Patient no. & $\mathrm{ng} / \mu \mathrm{l}$ & $\mathrm{A}_{260 / 280}$ & $\mathrm{~A}_{260 / 230}$ & Volume $(\mu \mathrm{l})$ & $\begin{array}{l}\text { Total } \\
\text { RNA }(\mu \mathrm{g})\end{array}$ \\
\hline 1 & 151.6 & 2.02 & 1.98 & 30 & 4.548 \\
2 & 315 & 2.05 & 2.02 & 50 & 15.750 \\
3 & 545.3 & 2.05 & 2.18 & 30 & 16.359 \\
4 & 699 & 2.04 & 1.97 & 50 & 34.950 \\
5 & 138 & 1.90 & 1.87 & 30 & 4.140 \\
6 & 344.4 & 2.06 & 2.14 & 50 & 17.220 \\
\hline
\end{tabular}

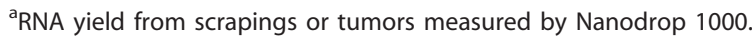

Another advantage of this method is to obtain fresh cancer cells for experimental purposes. By superficial scraping, the pathologist can provide this material without compromising
Table 3 DNA quality control ${ }^{\mathrm{a}}$

\begin{tabular}{lrrrcc}
\hline Patient no. & $\mathrm{ng} / \mu \mathrm{l}$ & $\mathrm{A}_{260 / 280}$ & $\mathrm{~A}_{260 / 230}$ & Volume $(\mu \mathrm{l})$ & Total DNA $(\mu \mathrm{g})$ \\
\hline 1 & 271.09 & 1.91 & 2.39 & 100 & 27.109 \\
2 & 26.40 & 1.92 & 3.58 & 100 & 2.640 \\
3 & 73.22 & 1.90 & 2.64 & 100 & 7.322 \\
4 & 78.15 & 1.94 & 2.32 & 100 & 7.815 \\
5 & 32.83 & 1.93 & 3.01 & 100 & 3.283 \\
6 & 102.55 & 1.96 & 2.64 & 100 & 10.255 \\
7 & 22.06 & 1.89 & 1.51 & 100 & 2.206 \\
\hline
\end{tabular}

${ }^{a}$ DNA quantification and estimated purity of snap frozen scrapings were performed using Nanodrop 1000.

the routine diagnostic procedure. The researcher can in turn expand these cells as cancer stem cells or differentiated cells for experimental studies. We show that CSC can be isolated 


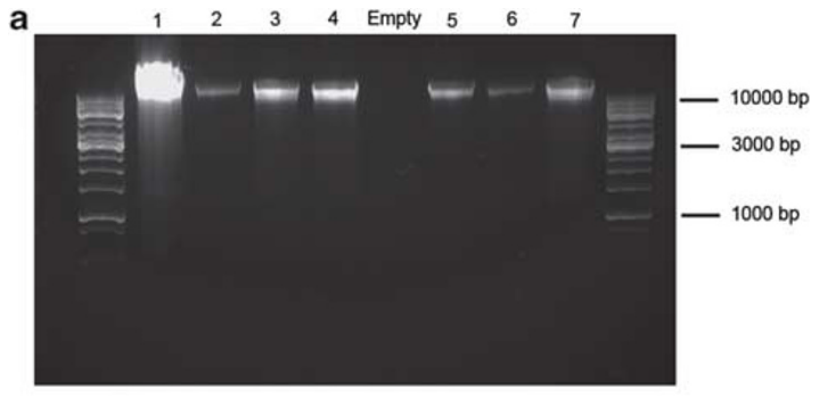

b

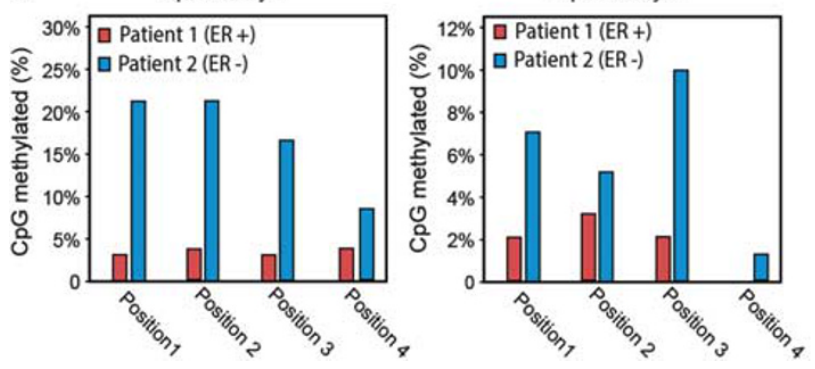

Figure 5 DNA quality and quantity control of scrapings from seven patients. (a) Genomic DNA integrity and purity shown as bands accessed by regular gel electrophoresis. (b) Estrogen receptor $\alpha(E R \alpha)$ methylation pattern of scraping correlates with the ER $\alpha$ expression of bulk tumor. DNA from the scrapings of two patients $(E R \alpha+(90 \%)$ and $E R \alpha-(0 \%))$ was used to analyze the $E R \alpha$ methylation status using pyrosequencing technique. Two $\mathrm{CpG}$ assays were used to cover eight $\mathrm{CpG}$ islands across the $E R \alpha$ promoter region. Methylation percentage for each site is represented in the graph.

in high frequency by superficial scraping. By growth in low serum-selective cell medium, we generate mammospheres with low risk of contamination from fibroblasts. The mammospheres that are phenotypically considered CSCs can then be differentiated to form adherent cancer cells.

To use snap frozen scraping material, it is important to guarantee the quality RNA and DNA from procured scrapings. Although RNA is easily degraded when extracted from small amount of material, intact RNA can be routinely isolated from snap frozen tissues according to previous studies. ${ }^{5,24}$ We compared the RNA quality of scrapings to that of the snap frozen tissue samples side by side. All the scraping samples exhibited clear ribosomal RNA peaks and the RIN values above 7 , similar to the tissue samples, indicating no major degradation. It should be pointed out that some studies have reported that RNA from samples with high proportions of epithelium showed higher RIN scores compared with stroma-rich samples. ${ }^{25}$ Indeed, we observed slightly better RNA peaks $(18 \mathrm{~S} / 28 \mathrm{~S})$ in our scrapings compared with tissue samples without observing much difference in the RIN values.

In addition, to confirm whether mRNA expression pattern of the scrapings can represent their parental tumors, we selected two genes as a reference, $E R \alpha$ and $P R$. As expected, $E R \propto$ mRNA expression level varied across samples. $P R$
mRNA, however, varied in one patient, perhaps, owing to a high epithelial component. To further investigate the cell components within scrapings, we examined fibroblast gene PDGFR $\beta$, myofibroblast gene $\alpha$-SMA and epithelial gene EPCAM expressions. Interestingly, of the three patients examined, all expressed high levels of EpCAM and low levels of $\alpha$-SMA and PDGFR $\beta$ compared with the tissue samples, indicating an enrichment of the epithelial fraction by scrapings as discussed above. Taken together, our results provide evidence that stable mRNA can be isolated from scrapings and used for gene expression studies.

NGS technologies require good genomic integrity for reliable analysis. Further, we assessed DNA quality of snap frozen scrapings from seven patients. Except a small amount of RNA contamination, avoidable by subsequent RNAse treatment, the quantity and quality of purified DNA is sufficient for DNA exome sequencing.

Apart from the usage of gene expression and DNA sequencing techniques, we have also shown that DNA from scrapings retained the methylation status of genes. In recent years, studies at the epigenetic level have appeared as promising methods to predict therapy response. Several researchers have shown that $\mathrm{ER} \alpha$ negativity is a consequence of $E R \propto$ promoter methylation..$^{26,27}$ We sought to investigate if the DNA extracted from scraping was of sufficient quality and quantity for DNA methylation analysis. After analyzing two patient's DNA, one ER $\alpha+$ and one ER $\alpha$ - , we observed $E R \alpha$ promoter methylation in the ER $\alpha$ - patient. Although more thorough investigations are needed, our results indicate that the DNA from scraping material can be used for studying genome methylation patterns.

CSCs derived form human breast cancer samples is a valuable resource for translational cancer research. However, current isolation protocols are based on fresh biopsies and are seldom available for research purposes. Although all scientists are aware of their drawbacks, cell lines have to be used as a replacement for CSC studies. As our protocol efficiently generates cancer cells even from small tumors, pathologists should now be able to provide this material without risking diagnostics.

In summary, we show that superficial scraping material can be used as an effective source for both biobanking and isolation and propagation of CSCs in parallel.

Supplementary Information accompanies the paper on the Laboratory Investigation website (http://www.laboratoryinvestigation.org)

\section{ACKNOWLEDGMENTS}

We thank Susanne Agartz for the management of patient materials, and Simon Sundling for the technical assistance in this study. Swedish Research Council (VR), the Breast Cancer Theme Center (BRECT), the Swedish Cancer Society, the research funds at Radiumhemmet, Swedish Society for Medical Research (SSMF) provided funding for experiments in the study.

\section{DISCLOSURE/CONFLICT OF INTEREST}

The authors declare no conflict of interest. 
1. Sorlie T, Perou CM, Tibshirani R, et al. Gene expression patterns of breast carcinomas distinguish tumor subclasses with clinical implications. Proc Nat Acad Sci USA 2001;98:10869-10874.

2. Goldhirsch A, Winer EP, Coates AS, et al. Personalizing the treatment of women with early breast cancer: highlights of the St Gallen International Expert Consensus on the Primary Therapy of Early Breast Cancer 2013. Ann Oncol 2013, 24:2206-2223.

3. Morente $\mathrm{MM}$, Mager $\mathrm{R}$, Alonso $\mathrm{S}$, et al. TuBaFrost 2: standardising tissue collection and quality control procedures for a European virtual frozen tissue bank network. Eur J Cancer 2006;42:2684-2691.

4. Mager SR, Oomen MH, Morente MM, et al. Standard operating procedure for the collection of fresh frozen tissue samples. Eur J Cancer 2007;43:828-834.

5. Micke $\mathrm{P}$, Ohshima $\mathrm{M}$, Tahmasebpoor $\mathrm{S}$, et al. Biobanking of fresh frozen tissue: RNA is stable in nonfixed surgical specimens. Lab Invest 2006:86:202-211.

6. Sjoblom $T$, Jones $S$, Wood LD, et al. The consensus coding sequences of human breast and colorectal cancers. Science 2006;314:268-274.

7. Ericsson C, Franzen B, Nister M. Frozen tissue biobanks. Tissue handling, cryopreservation, extraction, and use for proteomic analysis. Acta Oncol 2006;45:643-661.

8. Frampton GM, Fichtenholtz A, Otto $G A$, et al. Development and validation of a clinical cancer genomic profiling test based on massively parallel DNA sequencing. Nat Biotechnol 2013;31:1023-1031.

9. Botling J, Micke P. Fresh frozen tissue: RNA extraction and quality control. Methods Mol Biol 2011;675:405-413.

10. Mathot $L$, Lindman $M$, Sjoblom T. Efficient and scalable serial extrac tion of DNA and RNA from frozen tissue samples. Chem Commun (Camb) 2011;47:547-549.

11. Chen JL, Espinosa I, Lin AY, et al. Stromal responses among common carcinomas correlated with clinicopathologic features. Clin Cancer Res 2013;19:5127-5135.

12. Chin L, Hahn WC, Getz G, et al. Making sense of cancer genomic data. Genes Dev 2011;25:534-555.

13. Holley $T$, Lenkiewicz E, Evers L, et al. Deep clonal profiling of formalin fixed paraffin embedded clinical samples. PLoS One 2012;7:e50586.

14. Marsden CG, Wright MJ, Pochampally R, et al. Breast tumor-initiating cells isolated from patient core biopsies for study of hormone action. Methods Mol Biol 2009;590:363-375.
15. Beck B, Blanpain C. Unravelling cancer stem cell potential. Nat Rev Cancer 2013;13:727-738.

16. Al-Haij M Wicha MS, Benito-Hernandez $A$, et al. Prospective identification of tumorigenic breast cancer cells. Proc Natl Acad Sci USA 2003;100:3983-3988.

17. Ginestier C, Hur MH, Charafe-Jauffret E, et al. ALDH1 is a marker of normal and malignant human mammary stem cells and a predictor of poor clinical outcome. Cell Stem Cell 2007;1:555-567.

18. Pece S, Tosoni D, Confalonieri S, et al. Biological and molecular heterogeneity of breast cancers correlates with their cancer stem cell content. Cell 2010;140:62-73.

19. Buess M, Rajski M, Vogel-Durrer BM, et al. Tumor-endothelial interaction links the CD44( +)/CD24( - ) phenotype with poor prognosis in early-stage breast cancer. Neoplasia 2009;11:987-1002.

20. Ponti D, Costa A, Zaffaroni N, et al. Isolation and in vitro propagation of tumorigenic breast cancer cells with stem/progenitor cell properties. Cancer Res 2005;65:5506-5511.

21. Grimshaw MJ, Cooper L, Papazisis K, et al. Mammosphere culture of metastatic breast cancer cells enriches for tumorigenic breast cancer cells. Breast Cancer Res 2008;10:R52.

22. Hass R, Bertram C. Characterization of human breast cancer epithelial cells (HBCEC) derived from long term cultured biopsies. J Exp Clin Cancer Res 2009;28:127.

23. Emerman JT, Wilkinson DA. Routine culturing of normal, dysplastic and malignant human mammary epithelial cells from small tissue samples. In Vitro Cell Dev Biol 1990;26:1186-1194.

24. Bao WG, Zhang $X$, Zhang JG, et al. Biobanking of fresh-frozen human colon tissues: impact of tissue ex vivo ischemia times and storage periods on RNA quality. Ann Surg Oncol 2013;20: 1737-1744.

25. Bertilsson $\mathrm{H}$, Angelsen $\mathrm{A}$, Viset $\mathrm{T}$, et al. RNA quality in fresh frozen prostate tissue from patients operated with radical prostatectomy. Scan J Clin Lab Invest 2010:70:45-53.

26. Wei J, Han B, Mao XY, et al. Promoter methylation status and expression of estrogen receptor alpha in familial breast cancer patients. Tumour Biol 2012;33:413-420.

27. Giacinti L, Claudio PP, Lopez M, et al. Epigenetic information and estrogen receptor alpha expression in breast cancer. Oncologist 2006;11:1-8 